Bull. Austral. Math. Soc.

$41 \mathrm{~A} 05,41 \mathrm{~A} 10,41 \mathrm{~A} 25$

VOL. $62(2000) \quad[357-368]$

\title{
ON LAGRANGE INTERPOLATION WITH EQUALLY SPACED NODES
}

\author{
Michael Revers
}

\begin{abstract}
A well-known result due to S.N. Bernstein is that the sequence of Lagrange interpolation polynomials for $|x|$ at equally spaced nodes in $[-1,1]$ diverges everywhere, except at zero and the end-points. In this paper we present a quantitative version concerning the divergence behaviour of the Lagrange interpolants for $|x|^{3}$ at equidistant nodes. Furthermore, we present the exact rate of convergence for the interpolatory parabolas at the point zero.
\end{abstract}

\section{INTRODUCTION}

Let $f$ be a function defined on $[-1,1]$ and denote by $L_{n}(f,$.$) the Lagrange interpo-$ lating polynomial of degree at most $n$ to $f$ associated with the equidistant nodes

$$
x_{j}^{(n)}:=-1+\frac{2 j}{n}, \quad(j=0,1, \ldots, n, n=1,2, \ldots) .
$$

As is well known, the assumption that $f$ is continuous on $[-1,1]$ does not guarantee that $L_{n}(f,$.$) converges to f$ everywhere in $[-1,1]$ as $n \rightarrow \infty$. In 1918, Bernstein [1] showed that for $f(x)=|x|$, the sequence $\left\{L_{n}\left(f, x_{0}\right)\right\}_{n \geqslant 1}$ diverges for every point $x_{0} \in[-1,1]$ different from $-1,0,1$. The points -1 and 1 are interpolation points for every polynomial $L_{n}(f,$.$) and therefore L_{n}(|x|,-1)=L_{n}(|x|, 1)=1$. For the point zero it is proved in Natanson $[8, \mathrm{pp} .30-35]$ that

$$
\lim _{n \rightarrow \infty} L_{n}(|x|, 0)=0 \text {. }
$$

This result is due to D.L. Berman in 1939 and S.M. Lozinskii showed more exactly that $\left|L_{n}(|x|, 0)\right|<C / n$. A short survey on this topic is given in $[13, \mathrm{p} .285]$. There is a wide range of literature around Bernstein's classical divergence result. For example, see $[2,3,7,9,10,11,12,14]$. An extension of Bernstein's result is given in [10]:

TheOREM 1 . Let $0<\alpha \leqslant 1$. Then

$$
\varlimsup_{n \rightarrow \infty}\left|L_{n}\left(|x|^{\alpha}, x_{0}\right)\right|=\infty, \quad \forall x_{0} \in(-1,1), x_{0} \neq 0 .
$$

Received 15th November, 1999

Copyright Clearance Centre, Inc. Serial-fee code: 0004-9727/00 \$A2.00+0.00. 
Theorem 1 informs us that the divergence behaviour is rather general and does not depend on the special characteristics of $|x|$. In 1990, Byrne, Mills and Smith, amplifying the classical result of Bernstein, showed that the rate of divergence of the sequence $\left\{L_{n}\left(|x|, x_{0}\right)\right\}_{n \geqslant 1}$ depends on the location of $x_{0}$ in $[-1,1]$. More precisely, in [3] they proved the following:

ThEOREM 2. Let $0<\left|x_{0}\right|<1$. Then

$$
\varlimsup_{n \rightarrow \infty} \frac{1}{n+1} \log \left|L_{n}\left(|x|, x_{0}\right)-\right| x_{0}||=\frac{1}{2}\left[\left(1+x_{0}\right) \log \left(1+x_{0}\right)+\left(1-x_{0}\right) \log \left(1-x_{0}\right)\right] .
$$

For further references, see also Li and Mohapatra [5]. An extension of Theorem 2 to Hermite-Fejér (HF) interpolation with equidistant nodes (but for a different $f$ ) is given in Mills and Smith [7]. The aim of this paper is to show that Theorem 2, which is concerned with the rate of divergence of Lagrange interpolation for $|x|$, is not an isolated phenomenon and thus can be extended to the following result.

\section{Results}

We shall prove the following:

THEOREM 3. Let $0<\left|x_{0}\right|<1$. Then

$$
\left.\varlimsup_{n \rightarrow \infty} \frac{1}{n+1} \log \left|L_{n}\left(|x|^{3}, x_{0}\right)-\right| x_{0}\right|^{3} \mid=\frac{1}{2}\left[\left(1+x_{0}\right) \log \left(1+x_{0}\right)+\left(1-x_{0}\right) \log \left(1-x_{0}\right)\right] .
$$

Furthermore, recalling the above mentioned results of D.L. Berman and S.M. Lozinskii, we establish the exact rate of convergence at the point zero. More precisely we shall prove:

ThEOREM 4. Let $n=2 m-1, m \in \mathbb{N}, m \geqslant 2$. Then

$$
\frac{2}{\pi} \frac{1}{n^{3}}\left[1+\frac{2}{n-2}\right] \leqslant\left|L_{n}\left(|x|^{3}, 0\right)\right| \leqslant \frac{2}{\pi} \frac{1}{n^{3}}\left[1+\frac{2}{n-2}\right]\left[1+\frac{2}{n-1}\right] .
$$

Before presenting the details for the proofs let us mention some interesting aspects concerned with the results so far demonstrated. Certainly, Theorem 2 and Theorem 3 are attractive in so far as they lead us to the following conjecture:

Conjecture 5. Let $\alpha \in \mathbb{R}_{+} \backslash 2 \mathbb{N}$ and $0<\left|x_{0}\right|<1$. Then

$$
\left.\varlimsup_{n \rightarrow \infty} \frac{1}{n+1} \log \left|L_{n}\left(|x|^{\alpha}, x_{0}\right)-\right| x_{0}\right|^{\alpha} \mid=\frac{1}{2}\left[\left(1+x_{0}\right) \log \left(1+x_{0}\right)+\left(1-x_{0}\right) \log \left(1-x_{0}\right)\right] .
$$

We point out that a careful investigation into the proof of Theorem 1 strongly indicates the truth of Conjecture 5, at least for the restricted case $\alpha \in(0,1]$. However, at the moment I do not see a way of furnishing a proof for Conjecture 5 based on the methods established in this paper. On the other hand, turning to Theorem 4 combined 
with the results of Berman and Lozinskii, no choice is left but to believe that the exact rate of convergence at the point zero for the interpolants of $|x|^{\alpha}$ is given by $O\left(n^{-\alpha}\right)$. This, if true, would match perfectly with the following result established in [11]:

THEOREM 6 . Let $m \in \mathbb{N}, n=2 m-1$ and $0 \leqslant \alpha \leqslant 1$. Then

$$
\frac{2}{\pi} \frac{1}{n^{\alpha}} \leqslant\left|L_{n}\left(|x|^{\alpha}, 0\right)\right| \leqslant \frac{1}{n^{\alpha}} .
$$

We briefly describe the organisation of this paper. In section 3 we introduce some preparatory work which is required later in the proof. The next sections 4 and 5 deal with estimates for the cases $n=2 m$ and $n=2 m-1$, respectively. Then we establish the proof of Theorem 3. The last section presents the proof of Theorem 4. We note that some ideas in this paper were motivated by the work of Byrne, Mills and Smith [3].

\section{Preliminaries}

We introduce the Pochhammer notation $(.)_{j}$, the generalised hypergeometric function ${ }_{p} F_{q}$, and we collect some important lemmata. For $a \in \mathbb{R}, j=0,1,2, \ldots$, we define $(a)_{j}$ by

$$
\begin{aligned}
& (a)_{0}=1, \\
& (a)_{j}=a(a+1) \cdots(a+j-1), \quad(j=1,2,3, \ldots) .
\end{aligned}
$$

The generalised hypergeometric function ${ }_{p} F_{q}$ is introduced by

$$
{ }_{p} F_{q}\left(\begin{array}{c}
\alpha_{1}, \alpha_{2}, \ldots, \alpha_{p} \\
\beta_{1}, \beta_{2}, \ldots, \beta_{q}
\end{array} \mid z\right)=\sum_{j=0}^{\infty} \frac{\left(\alpha_{1}\right)_{j}\left(\alpha_{2}\right)_{j} \cdots\left(\alpha_{p}\right)_{j}}{\left(\beta_{1}\right)_{j}\left(\beta_{2}\right)_{j} \cdots\left(\beta_{q}\right)_{j}} \frac{z^{j}}{j !} .
$$

Then one can easily establish the following identities.

LEMma 7. If not defined otherwise, let $m \in \mathbb{N}, k=0,1,2, \ldots, m$ and $j=$ $0,1,2, \ldots$ We have

(a) $(m+k+j) !=(m+k) !(1+k+m)_{j}$.

(b) For $j \leqslant m-k-1$ and $k \leqslant m-1$ we have $(m-k-j-1)$ ! = $(-1)^{j} \frac{(m-k-1) !}{(1+k-m)_{j}}$

(c) $(1+k-m)_{j}=0, \quad(j \geqslant m-k$ and $k \leqslant m-1)$.

(d) $j+k=k \frac{(1+k)_{j}}{(k)_{j}}, \quad(k=1,2, \ldots)$.

(e) For $k=0,1,2, \ldots$ we have $1+2 j+2 k=(1+2 k) \frac{(3 / 2+k)_{j}}{(1 / 2+k)_{j}}$.

For $k=0,1,2, \ldots$ and $x \in(0,1)$ we have 
(f) $\frac{k+m x}{j+k+m x}=\frac{(k+m x)_{j}}{(1+k+m x)_{j}}$,

(g) $\frac{1+2 k+(2 m-1) x}{1+2 j+2 k+(2 m-1) x}=\frac{((1-x) / 2+k+m x)_{j}}{((3-x) / 2+k+m x)_{j}}$.

LEMMA 8. We shall denote the gamma function by $\Gamma$ (.).

(a) Let $c \neq 0,-1,-2, \ldots$ and $c-a-b>0$. Then

$$
{ }_{2} F_{1}\left(\begin{array}{l|l}
a, b & 1 \\
c & 1
\end{array}\right)=\frac{\Gamma(c) \Gamma(c-a-b)}{\Gamma(c-a) \Gamma(c-b)} .
$$

(b) Let $s=d+e-a-b-c$ and $s \neq 0$. Then

$$
{ }_{3} F_{2}\left(\begin{array}{l|l}
a, b, c & 1 \\
d, e & 1
\end{array}\right)=\frac{\Gamma(d) \Gamma(e) \Gamma(s)}{\Gamma(a) \Gamma(s+b) \Gamma(s+c)}{ }_{3} F_{2}\left(\begin{array}{l}
d-a, e-a, s \\
s+b, s+c
\end{array} \mid 1\right) \text {. }
$$

(c) Let $n=0,1,2, \ldots$ and $\beta(z-n) \neq 0$ with $\beta, z \in \mathbb{R}$. Then

$$
{ }_{4} F_{3}\left(\begin{array}{c|c}
-n, \beta+1,1, z+2 \beta \\
n+2 \beta+1, \beta, 1-z
\end{array} \mid 1\right)=\frac{z(n+2 \beta)}{2 \beta(z-n)}
$$

The above mentioned formulas are well known. See, for example in [6], p.99 for assertion (a), p.104 for (b) and p.113 for (c). The next lemma establishes some identities for certain binomial sums in terms of hypergeometric functions.

LEMMA 9. Let $m \in \mathbb{N}$ and $x \in(0,1)$. For $k=1,2, \ldots, m$ we have

For $k=0,1, \ldots, m-1$ we have

(a) $\sum_{j=k}^{m}(-1)^{j-1}\left(\begin{array}{c}2 m \\ m+j\end{array}\right) j=(-1)^{k-1}\left(\begin{array}{c}2 m \\ m+k\end{array}\right) \frac{2 k-1}{2} \frac{m+k}{2 m-1}$,

(b) $\sum_{j=k}^{m}(-1)^{j-1}\left(\begin{array}{c}2 m \\ m+j\end{array}\right) \frac{1}{j+m x}$

$$
=(-1)^{k-1}\left(\begin{array}{c}
2 m \\
m+k
\end{array}\right) \frac{1}{k+m x}{ }_{3} F_{2}\left(\begin{array}{l}
k+m x, k-m, 1 \\
1+k+m x, 1+k+m
\end{array} \mid 1\right) .
$$

$$
\text { (c) } \begin{array}{r}
\sum_{j=k}^{m-1}(-1)^{j+1}\left(\begin{array}{c}
2 m-1 \\
m+j
\end{array}\right)(1+2 j)^{2}=(-1)^{k+1}\left(4 k^{2}-1\right)\left(\begin{array}{c}
2 m-1 \\
m+k
\end{array}\right) \frac{m+k}{2 m-3}, \\
\text { (d) } \begin{array}{r}
\sum_{j=k}^{m-1}(-1)^{j+1}\left(\begin{array}{c}
2 m-1 \\
m+j
\end{array}\right) \frac{1+2 j}{1+2 j+(2 m-1) x}=(-1)^{k}\left(\begin{array}{c}
2 m-1 \\
m+k
\end{array}\right) \\
\cdot\left[\frac{(2 m-1) x}{1+2 k+(2 m-1) x}{ }_{3} F_{2}\left(\begin{array}{c}
(1-x) / 2+k+m x, 1+k-m, 1 \\
(3-x) / 2+k+m x, 1+k+m
\end{array} \mid 1\right)\right. \\
\left.-\frac{m+k}{2 m-1}\right] .
\end{array}
\end{array}
$$


PROOF: The proof of Lemma 9 can be established by some elementary calculations which are based on the identities in Lemma 7 and 8 . For illustration, we present a proof of assertion (d). First, the left-hand side of (d) can be rewritten as

$$
\begin{aligned}
& \sum_{j=0}^{m-1-k}(-1)^{j+1+k}\left(\begin{array}{c}
2 m-1 \\
m+j+k
\end{array}\right) \\
& \quad-(2 m-1) x \sum_{j=0}^{m-1-k}\left(\begin{array}{c}
2 m-1 \\
m+j+k
\end{array}\right) \frac{(-1)^{j+1+k}}{1+2 j+2 k+(2 m-1) x} .
\end{aligned}
$$

We now apply Lemma $7(\mathrm{a}-\mathrm{c})$ and $(\mathrm{g})$ and calculate the latter quantity to be

$$
\begin{aligned}
& (-1)^{k+1}\left(\begin{array}{c}
2 m-1 \\
m+k
\end{array}\right)\left[{ }_{2} F_{1}\left(\begin{array}{c|c}
1+k-m, 1 \\
1+k+m
\end{array} \mid 1\right)\right. \\
& \left.-\frac{(2 m-1) x}{1+2 k+(2 m-1) x}{ }_{3} F_{2}\left(\begin{array}{c|c}
(1-x) / 2+k+m x, 1+k-m, 1 & 1 \\
(3-x) / 2+k+m x, 1+k+m & 1
\end{array}\right)\right] .
\end{aligned}
$$

Next, by applying Lemma 8(a), we are able to establish the right-hand side of Lemma $9(d)$.

Our next task is to find a presentation for the interpolating polynomials for $|x|^{\alpha}$ based on the equidistant nodes (1). To this end let $n \in \mathbb{N}, x_{j}^{(n)}$ to be defined from (1) and denote by

$$
l_{k}^{(n)}(x)=\frac{w(x)}{\left(x-x_{k}^{(n)}\right) w^{\prime}\left(x_{k}^{(n)}\right)}, \quad(k=0,1, \ldots, n)
$$

the Lagrange fundamental polynomials with $w(x)=\prod_{j=0}^{n}\left(x-x_{j}^{(n)}\right)$. From the well known Lagrange interpolating formula it follows that

$$
L_{n}\left(|x|^{\alpha}, x_{0}\right)=\sum_{j=0}^{n}\left|x_{j}^{(n)}\right|^{\alpha} l_{j}^{(n)}\left(x_{0}\right)=\sum_{j=0}^{n}\left|x_{j}^{(n)}\right|^{\alpha} \frac{w\left(x_{0}\right)}{\left(x_{0}-x_{j}^{(n)}\right) w^{\prime}\left(x_{j}^{(n)}\right)} .
$$

Then, calculating the polynomials $w\left(x_{0}\right)$ and $w^{\prime}\left(x_{j}^{(n)}\right)$ (following the method from Runck $\left[12\right.$, pp.56-57]) we establish for $\alpha=3, m=1,2, \ldots$ and all fixed $x_{0}$ in $[0,1]$ the formulas

(2) $\left.\left|L_{2 m}\left(|x|^{3}, x_{0}\right)-\right| x_{0}\right|^{3}|=| \frac{\sin \pi m x_{0}}{\pi} \mid \frac{\Gamma\left[1+m\left(1+x_{0}\right)\right] \Gamma\left[1+m\left(1-x_{0}\right)\right]}{(2 m) !}$

$$
\cdot \frac{x_{0}}{m^{2}}\left|m x_{0}\left(\begin{array}{c}
2 m \\
m
\end{array}\right)-2 \sum_{j=1}^{m}(-1)^{j-1}\left(\begin{array}{c}
2 m \\
m+j
\end{array}\right) \frac{j^{3}-\left(m x_{0}\right)^{3}}{j^{2}-\left(m x_{0}\right)^{2}}\right|
$$


and

(3)

$$
\begin{aligned}
\left.\left|L_{2 m-1}\left(|x|^{3}, x_{0}\right)-\right| x_{0}\right|^{3} \mid & =\left|\frac{\cos \pi(m-1 / 2) x_{0}}{\pi}\right| \frac{\Gamma\left[1+(m-1 / 2)\left(1+x_{0}\right)\right] \Gamma\left[1+(m-1 / 2)\left(1-x_{0}\right)\right]}{(2 m-1) !} \\
& \cdot \frac{4}{(2 m-1)^{3}}\left|\sum_{j=0}^{m-1}(-1)^{j}\left(\begin{array}{c}
2 m-1 \\
m+j
\end{array}\right)(1+2 j) \frac{(1+2 j)^{3}-\left[(2 m-1) x_{0}\right]^{3}}{(1+2 j)^{2}-\left[(2 m-1) x_{0}\right]^{2}}\right|
\end{aligned}
$$

Since the polynomials $L_{n}\left(|x|^{\alpha},.\right)$, as well as $|x|^{\alpha}$, are even functions, it is sufficient to restrict ourselves to the interval $(0,1)$. The essential analysis now depends on the estimates of the right-hand sides in (2) and (3). We approach this problem by separating the cases $n=2 m$ and $n=2 m-1$. In the next two sections we shall establish lower and upper estimates for the corresponding formulas (2) and (3).

\section{Estimates for the Even Case}

Let $n=2 m$ with $m \in \mathbb{N}$. For $x_{0} \in(0,1)$ we define

$$
S_{m}\left(x_{0}\right):=\frac{x_{0}}{m^{2}}\left[2 \sum_{j=1}^{m}(-1)^{j-1}\left(\begin{array}{c}
2 m \\
m+j
\end{array}\right) \frac{j^{3}-\left(m x_{0}\right)^{3}}{j^{2}-\left(m x_{0}\right)^{2}}-m x_{0}\left(\begin{array}{c}
2 m \\
m
\end{array}\right)\right] .
$$

A standard argument (combined with Lemma 9(a-b)) enables us to rewrite the right-hand side in (4) more concisely as

$$
\frac{x_{0}}{m}\left(\begin{array}{c}
2 m \\
m
\end{array}\right)\left[\frac{1}{2 m-1}+\frac{2}{1+m} \frac{\left(m x_{0}\right)^{2}}{1+m x_{0}}{ }_{3} F_{2}\left(\begin{array}{l|l}
1+m x_{0}, 1-m, 1 \\
2+m x_{0}, 2+m
\end{array} \mid 1\right)-x_{0}\right] .
$$

We embark now on our study of the properties of (5).

4.1. Lower Estimate To this end, we shall show that (5) is positive (for sufficiently large $m$ ) and tends to infinity for $m \rightarrow \infty$ somewhat less than $\left(\begin{array}{c}2 m \\ m\end{array}\right)$. We begin with a summation of the ${ }_{3} F_{2}$ function in (5) by applying Lemma $8(\mathrm{~b})$. With $a=1+m x_{0}$, $b=1-m, c=1, d=2+m x_{0}, e=2+m$ it follows that $s=2 m+1$. A simple observation establishes

$$
{ }_{3} F_{2}\left(\begin{array}{l|l}
1+m x_{0}, 1-m, 1 \\
2+m x_{0}, 2+m
\end{array} \mid 1\right)=\frac{1+m x_{0}}{1+2 m}{ }_{3} F_{2}\left(\begin{array}{l|l}
1,1+m-m x_{0}, 1+2 m \\
2+m, 2+2 m
\end{array} \mid 1\right) .
$$

From Lemma 8(b) we see that we can express the (finite) alternating series in the lefthand side of (6) as an infinite sum which consists only of positive terms. Again, by 
applying Lemma $8(\mathrm{~b})$ to the right-hand side of (6) we get with a new $s\left(s=1+m x_{0}\right)$

$$
\text { (7) } \begin{aligned}
{ }_{3} F_{2} & \left(\begin{array}{l|l}
1+m x_{0}, 1-m, 1 \\
2+m x_{0}, 2+m
\end{array}\right.
\end{aligned}
$$

Since ${ }_{3} F_{2}$ is a symmetric function in its arguments we may exchange the arguments $1+2 m$ and $1+m x_{0}$ on the right-hand side in (7). Now we apply the same procedure once again. With a new $s(s=1)$ we establish

$$
{ }_{3} F_{2}\left(\begin{array}{l|l}
1+m x_{0}, 1-m, 1 \\
2+m x_{0}, 2+m
\end{array} \mid 1\right)=\frac{1+m}{1+2 m}{ }_{3} F_{2}\left(\begin{array}{c}
1+m+m x_{0}, 1,1 \\
2+m x_{0}, 2+2 m
\end{array} \mid 1\right) .
$$

Now, armed with expression (8), we can give an appropriate lower estimate. By rewriting ${ }_{3} F_{2}$ in terms of Pochhammer symbols we establish

$$
{ }_{3} F_{2}\left(\begin{array}{l|l}
1+m x_{0}, 1-m, 1 \\
2+m x_{0}, 2+m
\end{array} \mid 1\right) \geqslant \frac{1+m}{1+2 m} \sum_{j=0}^{3} \frac{\left(1+m+m x_{0}\right)_{j}(1)_{j}}{\left(2+m x_{0}\right)_{j}(2+2 m)_{j}} .
$$

We combine estimate (9) and formula (5). By a careful, but absolute elementary calculation, one shows that

$$
S_{m}\left(x_{0}\right) \geqslant\left(\begin{array}{c}
2 m \\
m
\end{array}\right) \frac{1}{m^{4}}\left(\frac{x_{0}}{27}\right)^{3}, \quad \forall m \geqslant 170 .
$$

We point out that the reader may, if he wishes, easily find comparable estimates to (10). However, it is not to hard to see that the upper summation index 3 in (9) may not be replaced by a smaller index to give a result which is comparable to (10).

4.2. UPPER Estimate To proceed further, we turn again to (4) and (5), to give an appropriate upper estimate for $S_{m}\left(x_{0}\right)$. From $(6)$ a standard argument leads to the following estimate given by

$$
{ }_{3} F_{2}\left(\begin{array}{l|l}
1+m x_{0}, 1-m, 1 \\
2+m x_{0}, 2+m
\end{array} \mid 1\right) \leqslant \frac{1+m x_{0}}{1+2 m}{ }_{2} F_{1}\left(\begin{array}{l|l}
1+m-m x_{0}, 1 \\
2+m
\end{array} \mid 1\right) .
$$

Then, by applying Lemma $8(a)$ to (11), we are able to estimate (5) for all $m \in \mathbb{N}$ by

$$
S_{m}\left(x_{0}\right) \leqslant \frac{x_{0}}{m}\left(\begin{array}{c}
2 m \\
m
\end{array}\right)\left[\frac{1}{2 m-1}+\frac{2 m x_{0}}{1+2 m}-x_{0}\right] \leqslant\left(\begin{array}{c}
2 m \\
m
\end{array}\right) \frac{x_{0}}{m} \frac{1}{2 m-1} .
$$

Combining (10), (12) and (2) we obtain

$$
\begin{aligned}
& \left|\frac{\sin \pi m x_{0}}{\pi}\right| \frac{\Gamma\left[1+m\left(1+x_{0}\right)\right] \Gamma\left[1+m\left(1-x_{0}\right)\right]}{\Gamma(1+m) \Gamma(1+m)} \frac{1}{m^{4}}\left(\frac{x_{0}}{27}\right)^{3} \\
& \quad \leqslant\left.\left|L_{2 m}\left(|x|^{3}, x_{0}\right)-\right| x_{0}\right|^{3} \mid \\
& \quad \leqslant\left|\frac{\sin \pi m x_{0}}{\pi}\right| \frac{\Gamma\left[1+m\left(1+x_{0}\right)\right] \Gamma\left[1+m\left(1-x_{0}\right)\right]}{\Gamma(1+m) \Gamma(1+m)} \frac{1}{2 m-1} \frac{x_{0}}{m},
\end{aligned}
$$


where both inequalities are valid for $x_{0} \in(0,1)$ and $m \geqslant 170$. Of course, we may extend (13) to all $x_{0} \in[0,1]$.

\section{Estimates for the Odd Case}

Let $n=2 m-1$ with $m \in \mathbb{N}$. As before, we define for $x_{0} \in(0,1)$

$$
\begin{aligned}
T_{m}\left(x_{0}\right):=\frac{4}{(2 m-1)^{3}} \sum_{j=0}^{m-1}\left[(-1)^{j+1}\left(\begin{array}{c}
2 m-1 \\
m+j
\end{array}\right)(1+2 j)\right. & \left.\cdot \frac{(1+2 j)^{3}-\left[(2 m-1) x_{0}\right]^{3}}{(1+2 j)^{2}-\left[(2 m-1) x_{0}\right]^{2}}\right] .
\end{aligned}
$$

Following the procedure for the even case (and applying Lemma $9(\mathrm{c}-\mathrm{d})$ ) we rewrite (14) more concisely as

$$
\begin{aligned}
& T_{m}\left(x_{0}\right)=\frac{4}{(2 m-1)^{3}}\left(\begin{array}{c}
2 m-1 \\
m
\end{array}\right)\left[\frac{m}{2 m-3}+\frac{\left[(2 m-1) x_{0}\right]^{3}}{1+(2 m-1) x_{0}}\right. \\
&\left.\cdot{ }_{3} F_{2}\left(\begin{array}{l}
\left(1-x_{0}\right) / 2+m x_{0}, 1-m, 1 \\
\left(3-x_{0}\right) / 2+m x_{0}, 1+m
\end{array} \mid 1\right)-(2 m-1) m x_{0}^{2}\right]
\end{aligned}
$$

5.1. Lower Estimate For the ${ }_{3} F_{2}$ function in (15) we apply a similar triplicate summation as in the corresponding subsection for the even case. We calculate

$$
\begin{aligned}
{ }_{3} F_{2}\left(\begin{array}{c|c}
\left(1-x_{0}\right) / 2+m x_{0}, 1 & -m, 1 \\
\left(3-x_{0}\right) / 2+m x_{0}, 1 & +m
\end{array} \mid 1\right) \\
=\frac{1}{2}{ }_{3} F_{2}\left(\begin{array}{c}
\left(1-x_{0}\right) / 2+m+m x_{0}, 1,1 \\
\left(3-x_{0}\right) / 2+m x_{0}, 1+2 m
\end{array}\right) \\
\geqslant \frac{1}{2} \sum_{j=0}^{4} \frac{\left(\left(1-x_{0}\right) / 2+m+m x_{0}\right)_{j}(1)_{j}}{\left(\left(3-x_{0}\right) / 2+m x_{0}\right)_{j}(1+2 m)_{j}} .
\end{aligned}
$$

Let us remark that the upper summation index 4 in the last expression of (16) is the smallest possible which works. Now, inserting (16) in (15), one establishes after a tedious calculation the following estimate:

$$
T_{m}\left(x_{0}\right) \geqslant\left(\begin{array}{c}
2 m-1 \\
m
\end{array}\right) \frac{4 x_{0}^{3}}{729 m^{2}} \frac{1}{(2 m-1)^{3}}, \quad m \geqslant \max \left(\frac{1}{x_{0}^{2}}, 744\right) .
$$

5.2. UPPER Estimate By a routine observation one checks that for $x_{0} \in(0,1), m \in \mathbb{N}$, $j=0,1, \ldots$ the following inequality

$$
\frac{\left(\left(1-x_{0}\right) / 2+m+m x_{0}\right)_{j}(1)_{j}}{\left(\left(3-x_{0}\right) / 2+m x_{0}\right)_{j}} \leqslant(m)_{j}
$$


holds, from which we conclude that

(19) ${ }_{3} F_{2}\left(\begin{array}{c|c}\left(1-x_{0}\right) / 2+m+m x_{0}, 1,1 & 1 \\ \left(3-x_{0}\right) / 2+m x_{0}, 1+2 m & 1\end{array}\right)$

$$
\leqslant \sum_{j=0}^{\infty} \frac{(m)_{j}}{(1+2 m)_{j}}={ }_{2} F_{1}\left(\begin{array}{l|l}
m, 1 \\
1+2 m
\end{array} \mid 1\right)=2,
$$

where we have used Lemma 8(a) for the last equality. Combining (15), the summation in (16) and (19), it is easy to establish (for all $m \in \mathbb{N}, x_{0} \in(0,1)$ )

$$
T_{m}\left(x_{0}\right) \leqslant\left(\begin{array}{c}
2 m-1 \\
m
\end{array}\right) \frac{8}{2 m-1} .
$$

Combining (17), (20) and (3) we derive our second substantial result. We have

$$
\begin{array}{r}
\left|\frac{\cos \pi(m-1 / 2) x_{0}}{\pi}\right| \frac{4 x_{0}^{3}}{729 m} \frac{1}{(2 m-1)^{3}} \\
\frac{\Gamma\left[1+(m-1 / 2)\left(1+x_{0}\right)\right] \Gamma\left[1+(m-1 / 2)\left(1-x_{0}\right)\right]}{\Gamma(1+m) \Gamma(1+m)} \\
\leqslant\left.\left|L_{2 m-1}\left(|x|^{3}, x_{0}\right)-\right| x_{0}\right|^{3} \mid \\
\leqslant\left|\frac{\cos \pi(m-1 / 2) x_{0}}{\pi}\right| \frac{8 m}{2 m-1} \\
\frac{\Gamma\left[1+(m-1 / 2)\left(1+x_{0}\right)\right] \Gamma\left[1+(m-1 / 2)\left(1-x_{0}\right)\right]}{\Gamma(1+m) \Gamma(1+m)},
\end{array}
$$

where both inequalities are valid for $x_{0} \in(0,1)$ and $m \geqslant \max \left(\left(1 / x_{0}^{2}\right), 744\right)$. Of course, we may extend this result to $x_{0} \in(0,1]$.

\section{Proof of TheOREM 3}

With the results (13) and (21) at our disposal, we are able to enter into the main proof. We begin with the right-hand side in (21). An easy computation reveals that, for all sufficiently large $m$, we may write

$$
\begin{aligned}
& \left.\frac{1}{m} \log \left|L_{2 m-1}\left(|x|^{3}, x_{0}\right)-\right| x_{0}\right|^{3} \mid \\
& \quad \leqslant \frac{1}{m} \log \frac{\Gamma\left[1+(m-1 / 2)\left(1+x_{0}\right)\right] \Gamma\left[1+(m-1 / 2)\left(1-x_{0}\right)\right]}{\Gamma(1+m) \Gamma(1+m)}+O\left(\frac{1}{m}\right) .
\end{aligned}
$$

Now, as $m \rightarrow \infty$, we may apply the asymptotic expansion for $\log \Gamma(x)$ (see, for example, [6, p.31])

$$
\log \Gamma(x)=\left(x-\frac{1}{2}\right) \log x-x+\frac{1}{2} \log 2 \pi+O\left(\frac{1}{x}\right), \quad x \rightarrow \infty,
$$


to (22) and we obtain (by some calculations)

$$
\begin{aligned}
& \left.\frac{1}{m} \log \left|L_{2 m-1}\left(|x|^{3}, x_{0}\right)-\right| x_{0}\right|^{3} \mid \\
& \leqslant \frac{1 / 2+(m-1 / 2)\left(1+x_{0}\right)}{m} \log \frac{1+(m-1 / 2)\left(1+x_{0}\right)}{1+m} \\
& \quad+\frac{1 / 2+(m-1 / 2)\left(1-x_{0}\right)}{m} \log \frac{1+(m-1 / 2)\left(1-x_{0}\right)}{1+m}+O\left(\frac{\log m}{m}\right) .
\end{aligned}
$$

Then, as $m \rightarrow \infty$ in (23), we obtain

$$
\begin{aligned}
\varlimsup_{m \rightarrow \infty} \frac{1}{2 m} \log \mid L_{2 m-1}\left(|x|^{3}, x_{0}\right)- & \left|x_{0}\right|^{3} \mid \\
& \leqslant \frac{1}{2}\left[\left(1+x_{0}\right) \log \left(1+x_{0}\right)+\left(1-x_{0}\right) \log \left(1-x_{0}\right)\right] .
\end{aligned}
$$

Employing similar arguments to the right-hand side of (13) implies that

$$
\begin{aligned}
\varlimsup_{m \rightarrow \infty} \frac{1}{2 m+1} \log \mid L_{2 m}\left(|x|^{3}, x_{0}\right) & -\left|x_{0}\right|^{3} \mid \\
& \leqslant \frac{1}{2}\left[\left(1+x_{0}\right) \log \left(1+x_{0}\right)+\left(1-x_{0}\right) \log \left(1-x_{0}\right)\right] .
\end{aligned}
$$

Now we turn to the left-hand side in (21). By passing to an appropriate subsequence we can establish the following

LEMMA 10. For every fixed $x_{0} \in(0,1)$ we can find two small positive numbers $\varepsilon_{1}$ and $\varepsilon_{2}$ and increasing subsequences $\left\{m_{j}^{(1)}\right\}_{j \geqslant 1}$ and $\left\{m_{j}^{(2)}\right\}_{j \geqslant 1}$ of positive integers such that for all $j=1,2, \ldots$ the following assertions hold:

$$
\begin{array}{r}
\left|\frac{\sin \pi m_{j}^{(1)} x_{0}}{\pi}\right| \geqslant \varepsilon_{1}\left(x_{0}\right)>0, \\
\left|\frac{\cos \pi\left(m_{j}^{(2)}-1 / 2\right) x_{0}}{\pi}\right| \geqslant \varepsilon_{2}\left(x_{0}\right)>0 .
\end{array}
$$

Proof: We establish only assertion (a), since the proof of (b) is very similar. We consider two cases:

CASE A. $x_{0} \in \mathbb{Q}$. We write $x_{0}$ in the form $a / b$ with $a, b \in \mathbb{N}$, and $(a, b)=1$. It is easy to give an explicit subsequence that works. For instance, the subsequence defined by $m_{j}^{(1)}=j b+1, j=1,2, \ldots$ will establish this case.

CASE B. $x_{0} \in \mathbb{R} \backslash \mathbb{Q}$. Then the sequence $\left\{\left|\sin \pi m x_{0}\right|\right\}_{m \geqslant 1}$ is dense in the interval $[0,1]$. This follows from the continuity of sin and the well known fact that the Kronecker sequence $\left\{m x_{0} \bmod 1\right\}_{m \geqslant 1}$ is dense in $[0,1]$. Of course, this sequence is uniformly distributed modulo 1 (see [4, p.8]). From this observation it follows at once that, for an appropriate small $\varepsilon_{1}>0$, we may select an increasing subsequence of positive integers such that the assertion holds in this case. 
Combining Lemma 10 with the left-hand side of (21) establishes

$$
\begin{aligned}
& \left.\frac{1}{m_{j}^{(2)}} \log \left|L_{2 m_{j}^{(2)}-1}\left(|x|^{3}, x_{0}\right)-\right| x_{0}\right|^{3} \mid \\
& \geqslant \frac{1}{m_{j}^{(2)}} \log \frac{\Gamma\left[1+\left(m_{j}^{(2)}-1 / 2\right)\left(1+x_{0}\right)\right] \Gamma\left[1+\left(m_{j}^{(2)}-1 / 2\right)\left(1-x_{0}\right)\right]}{\Gamma\left(1+m_{j}^{(2)}\right) \Gamma\left(1+m_{j}^{(2)}\right)} \\
& +O\left(\frac{\log m_{j}^{(2)}}{m_{j}^{(2)}}\right),
\end{aligned}
$$

where the estimate is only valid for sufficiently large integers $m_{j}^{(2)}$. As before, the righthand side of (26) tends to

$$
\left(1+x_{0}\right) \log \left(1+x_{0}\right)+\left(1-x_{0}\right) \log \left(1-x_{0}\right),
$$

as $m_{j}^{(2)} \rightarrow \infty$. Thus we arrive at the conclusion that

$$
\begin{aligned}
\varlimsup_{m \rightarrow \infty} \frac{1}{2 m} \log \mid L_{2 m-1}\left(|x|^{3}, x_{0}\right)- & \left|x_{0}\right|^{3} \mid \\
& \geqslant \frac{1}{2}\left[\left(1+x_{0}\right) \log \left(1+x_{0}\right)+\left(1-x_{0}\right) \log \left(1-x_{0}\right)\right] .
\end{aligned}
$$

To finish the proof, we combine (24), (25) and (27) to establish Theorem 3.

\section{Proof of Theorem 4}

Combing Lemma 9(c) and formula (3) (with $x_{0}=0$ ) it follows at once that

$$
\begin{aligned}
\left|L_{2 m-1}\left(|x|^{3}, 0\right)\right| & =\frac{1}{\pi} \frac{\Gamma(m+1 / 2) \Gamma(m+1 / 2)}{\Gamma(2 m)} \frac{4}{(2 m-1)^{3}}\left(\begin{array}{c}
2 m-1 \\
m
\end{array}\right) \frac{m}{2 m-3} \\
& =\frac{2}{\pi} \frac{\Gamma(m+1 / 2) \Gamma(m-1 / 2)}{\Gamma(m) \Gamma(m)} \frac{2 m-1}{2 m-3} \frac{1}{(2 m-1)^{3}} .
\end{aligned}
$$

Recalling the log-convexity of the gamma function, a standard argument shows that for $m \in \mathbb{N}, m \geqslant 2$ and $0 \leqslant x_{0} \leqslant 1$ the function

$$
f_{m}\left(x_{0}\right)=\frac{\Gamma\left(m+x_{0}\right) \Gamma\left(m-x_{0}\right)}{\Gamma(m) \Gamma(m)}
$$

is increasing (in $x_{0}$ ). Thus we have the estimates

$$
1 \leqslant \frac{\Gamma(m+1 / 2) \Gamma(m-1 / 2)}{\Gamma(m) \Gamma(m)} \leqslant 1+\frac{1}{m-1}, \quad m \geqslant 2 .
$$

Combining (30) and (28) establishes Theorem 4 and so we are done. 


\section{REFERENCES}

[1] S. Bernstein, 'Quelques remarques sur l'interpolation', Math. Ann. 79 (1918), 1-12.

[2] L. Brutman and E. Passow, 'On the Divergence of Lagrange interpolation to $|x|$ ', $J$. Approx. Theory 81 (1995), 127-135.

[3] G. Byrne, T.M. Mills and S.J. Smith, 'On Lagrange interpolation with equidistant nodes', Bull. Austral. Math. Soc. 42 (1990), 81-89.

[4] L. Kuipers and H. Niederreiter, Uniform distribution of sequences (John Wiley, New York, 1974).

[5] X. Li and R.N. Mohapatra, 'On the divergence of Lagrange interpolation with equidistant nodes', Proc. Amer. Math. Soc. 118 (1993), 1205-1212.

[6] Y.L. Luke, The special functions and their approximations, Vol. I (Academic Press, New York, 1969).

[7] T.M. Mills and S.J. Smith, 'On the divergence of Hermite-Fejér type interpolation with equidistant nodes', Bull. Austral. Math. Soc. 49 (1994), 101-110.

[8] I.P. Natanson, Constructive function theory, Vol. III (Frederick Ungar, New York, 1965).

[9] M. Revers, 'On the approximation of certain functions by interpolating polynomials', Bull. Austral. Math. Soc. 58 (1998), 505-512.

[10] M. Revers, 'The divergence of Lagrange interpolation for $|x|^{\alpha}$ at equidistant nodes', $J$. Approx. Theory 103 (2000), 269-280.

[11] M. Revers, 'On Lagrange interpolatory parabolas to $|x|^{\alpha}$ at equally spaced nodes', Arch. Math 74 (2000), 385-391.

[12] P.O. Runck, 'Über Konvergenzfragen bei Polynominterpolation mit äquidistanten Knoten I', J. Reine Angew. Math. 208 (1961), 51-69.

[13] Bl. Sendov and A. Andreev, 'Approximation and interpolation theory', in Handbook of numerical analysis, Vol. III, (P.G. Ciarlet and J.L. Lions, Editors) (North Holland, Amsterdam, 1994).

[14] R.S. Varga and A.J. Carpenter, 'On the Bernstein Conjecture in approximation theory', Constr. Approx. 1 (1985), 333-348.

Department of Mathematics

University of Salzburg

Hellbrunnerstrasse $\mathbf{3 4}$

A-5020 Slazburg

Austria

e-mail: Michael.Revers@sbg.ac.at 